\title{
Stc-M-injective and Stc-self-injective Modules
}

\section{Mahdi SalehNayef}

Department of Mathematics, College of education, University of Al-Mustansiriyah, Email: mahdisaleh773@uomustansiriyah.edu.iq.

\section{ABSTRACT}

In this paper we introduce the concepts Stc-M-injective and Stc-self-injective modules which are proper generalizations of $\mathrm{M}$-injective and self-injective respectively. Numerous properties of these generalizations are given. Moreover, we discussed the relationship between Stc-injectivity and Rc-injectivity, also the relation with C-injectivity.

Key words:St-closed submodules, rationally closed submodules, closed submodules, Stc-Minjective modules, M-rc-injective modules, c-self-injective, quasi-injective modules.

\section{Corresponding Author:Mahdi SalehNayef}

\section{INTRODUCTION}

Throughout this paper, we assume every ring $R$ to be an associative ring with identity and every $R$-modules are unitary right modules.

Let $M$ be an $R$-module, a module $A$ is called $M$-injective if for every submodule $T$ of $M$, any $R$ homomorphism from $T$ to $A$ can be extended to an $R$-homomorphism from $M$ to $A$. A module $A$ is called injective if it is $M$-injective for every $R$-module $M$. An $R$-module $A$ is called self(quasi)injective, if it is $A$-injective[9].

A submodule $T$ of an $R$-module $M$ is called rational in $M$ (briefly $T \leq_{r} M$ ), if for every $x, y \in M$ with $x \neq 0$ there exist $r \in R$ such that $y r \in T$ and $x r \neq 0$ [8]. It is well known that a nonzero submodule $T$ of $M$ is called essential (briefly $T \leq_{e} M$ ), if $T \cap K \neq(0)$ for each nonzero submodule $K$ of $M$ [9], and a nonzero submoduleT of $M$ is called semi-essential (briefly $\left.T \leq_{\text {sem }} M\right)$, if $T \cap P \neq(0)$ for each nonzero prime $R$-submodule $\mathrm{P}$ of $M$ [3]. Equivalently, a submodule $T$ of an R-module $M$ is called semi-essential if whenever $T \cap P=(0)$, then $P=(0)$ for every prime submodule $\mathrm{P}$ of $M$ [10], where a submodule $\mathrm{P}$ of $M$ is called prime, if whenever $r m \in P$ for $r \in R$ and $m \in M$, then either $m \in P$ or $r \in\left(P_{R}: M\right)$ [13].

An R-module $\mathrm{M}$ is called fully prime, if every proper submodule of $\mathrm{M}$ is a prime submodule [4] .So we have the following implications:

Rational submodules $\Rightarrow$ Essential submodules $\Rightarrow$ Semi-essential submodules. In general, neither of the converses of implication is holds. 
An R-module $\mathrm{M}$ is called monoform is every non-zero submodule of $\mathrm{M}$ is rational submodule of $\mathrm{M}$ [1]. An R-module $\mathrm{M}$ is called uniform(semi-uniform) is every non-zero submodule of $\mathrm{M}$ is essential(semi-essential) submodule of M[ 8][2]. So we have the following implications:

Monoform module $\Rightarrow$ Uniform module $\Rightarrow$ Semi-uniform module .

A submodule $H$ of an $R$-module $M$ is called rationally closed in $M$ (briefly $H \leq_{r c} M$ ), if $H$ has no proper rational extension in $M$,i.e if $H \leq_{r} K \leq M$ then $H=K$ [1].A submodule $H$ of $M$ is called closed submodule (briefly $H \leq_{c} M$ ), if $H$ has no proper essential extensions in M, i.e if $H \leq_{e} K \leq M$ then $H=K$ [6]. A submodule $H$ of an R-module $M$ is called St-closed if $H$ has no proper semi-essential extensions in $M$, i.e if $H \leq_{\text {sem }} K \leq M$ then $H=K$ [11].

Let $M$ be $R$-module. In [5] an $R$-module $A$ is called $M$-c-injective, if every $R$-homomorphism $\alpha: H \rightarrow A$, where $H$ is a closed submodule of $M$, can be extended to an $R$-homomorphism $\beta: M \rightarrow A$. An $R$-module $A$ is said to be self-c-injective if $A$ is $A$-c-injective.

An $R$-module $M$ is called C-quasi-injective if, any $R$-homomorphism $\varphi: H \rightarrow M$, where $H$ is a closed submodule of $M$, can be extended to some $\alpha \in \operatorname{End}(M)$ [14]. Obviously that, the concepts of self-c-injective and C-quasi-injective $R$-modules are the same.

In [2], Abbas, M. S., and Mahdi, S. N. introduced the concepts M-rc-injective and rc-quasiinjective modules. Let $M_{1}$ and $M_{2}$ be $R$-modules. Then $M_{2}$ is called $M_{1}-r c$-injective if every $R$ homomorphism $f: H \rightarrow M_{2}$, where $H$ is rationally closed submodule of $M_{1}$, can be extended to an $R$-homomorphism $g: M_{1} \rightarrow M_{2}$. An $R$-module $M$ is called $r c$-injective, if $M$ is $N$-rcinjective, for every $R$-module $N$. An $R$-module $M$ is called $r c$-quasi-injective or self- $r c-$ injective, if $M$ is $M-r c$-injective[2].

Thus we have the following implications:

Injective module $\Rightarrow$ quasi-injective module $\Rightarrow r c$-quasi-injective module $\Rightarrow c$-quasi-injective module.

In general, neither of the converses of implication is hold[2].

In this work, we introduce and study a proper generalization of $M$-injectivity and Self-injectivity, namely Stc- $M$-injective and Stc-self-injective modules respectively. Also, we will shows that the class of Stc-injectivity is a weak than the class of C-injectivity.

\section{SOMERESULTSON St-CLOSEDSUBMODULES}

In this section we introduce some results on St-closed submodule(briefly Stc-submodule) which needed in our work.

Definition 2.1: An R-module M is called STC-module if every submodule of M is Stc-submodule.

\section{Remarks and Examples 2.2:}

(1) $M=Z_{6}$ as Z-module is STC-module.

(2) $M=Z$ as Z-module is not STC-module, since a submodule $T=5 Z$ is not Stc-submodule of $Z$ since $T=5 Z$ is semi-essential submodule of $Z$ ( in fact $Z$ is uniform (and hence $Z$ is semiuniform). 
(3) Every field is not STC-module, since every filed has only one prime submodule which is $<0\rangle$ implies $\langle 0\rangle$ is semi-essential submodule. Therefor, $\langle 0\rangle$ is not Stc-submodule. For example, the Z-modules: $Z_{2}, Z_{3}, \ldots Z_{p}$ (where $\mathrm{p}$ is prime number) are not STC-modules.

(4) Follows [11], if D is a direct summand of an R-module $M$ then not necessary D is Stcsubmodule in $M$, for example: Consider the $Z$-module,

$Z_{36}=9 Z \oplus 4 Z$,it is clear that $9 Z$ is a direct summand of $Z_{36}$ but not Stc- submodule in $Z_{36}$

(5) Every St-closed submodule is rationally closed submodule.

Proof: Let $\mathrm{T}$ be an Stc-submodule of an R-module $M$. Then by [11,Remark(1.3)], T is closed submodule in $M$. Hence by [1], $\mathrm{T}$ is rationally closed submodule.

(6) the converse of (5), may not be true in general. For example a submodule $2 Z$ of $Z$-module $Z_{4}$ is rationally closed but not Stc-submodule, since $2 Z$ is semi-essential submodule in $Z_{4}$.

The following implication explain the relation between these submodules

St-closed submodules $\Rightarrow$ Closed submodules $\Rightarrow$ Rational closed submodules.

The converses of this implication may not be true in general.

Proposition2.3: Every submodule in STC-module is direct summand.

Proof: Suppose that $M$ be STC-module and $H$ be any a submodule of $M$. Then, by [definition (2.1)], $H$ is Stc-submodule of $M$ and hence by[11, Reamark(1.3)], $H$ is closed submodule of $\mathrm{M}$. This implies that, every submodule of $\mathrm{M}$ is closed submodule, thus by [9, P.139] , $\mathrm{H}$ is a direct summand in $\mathrm{M}$, that means, every submodule of $\mathrm{M}$ is direct summand.

An R-module $M$ is extending if and only if every closed submodule of $M$ is direct summand[6].

Corollary 2.4: Every STC-module is semisimple R-module( and hence extending module).

The converse of Corollary (2.4) may not be true in general. For example, consider $M=Z_{2}$ as Zmodule. It is well known that, $M$ is semi simple module( and hence extending module), but $M$ is not STC-module, since $<0>$ is not Stc-submodule of $Z_{2}$.

An R-module $M$ is called fully prime if every submodule of $M$ is prime[4].

Theorem 2.5:For fully prime R-module M. The following statements are equivalent:

(i) Mis STC-module.

(ii) Mis semi simple module.

Proof: (i) $\Rightarrow$ (ii) It is follows corollary (2.4).

(ii) $\Rightarrow$ (i) Suppose that, $M$ is semi simple module and $\mathrm{T}$ be any submodule of $M$. Then $\mathrm{T}$ is direct summand of $M$. It is well known that every direct summand is closed submodule, thus $\mathrm{T}$ is closed submodule in $M$. Since $M$ is fully prime R-module then by [11,Remark (1.8)], T is Stcsubmodule in $M$. Therefore, $M$ is STC-module.

Recall that a singular submodule defined by $Z(M)=\left\{m \in M: \operatorname{ann}(m) \leq_{e} R\right\} . M$ is called the singular module, if $Z(M)=M$, and $\mathrm{M}$ is called a nonsingular module, if $Z(M)=0$ [8]. 
In the following results we explain when the converses of the implication of remark $((2.2)(6))$ is true.

Firstly, we need the following lemmaswhich appeared in [11] and [1] respectively.

Lemma2.6: If an R-module $M$ is fully prime, then every nonzero closed submodule in $M$ is an Stclosed submodule.

Lemma2.7:If an R-module $M$ is non-singular, then every nonzero rationally closed submodule in $M$ is an closed submodule.

Proposition 2.8: Let Mbe non-singular fully prime R-module, and $T$ be non-zero submoduleof M. Then the following statements are equivalent:

(i) $T$ is St-closed submodule

(ii) $T$ is closed submodule

(iii) $T$ is rationally closed submodule.

Proof: $(i) \Leftrightarrow$ (ii)follows [11, the remarks (1.3)] and [Lemma (2.6)]

(ii) $\Leftrightarrow$ (iii)follows [1, definition (1.5)] and [Lemma (2.7)]

$(i) \Leftarrow($ iii)Suppose that $\mathrm{T}$ is rationally closed submodulein $\mathrm{M}$. Since $\mathrm{M}$ is non-singular then by [Lemma (2.7)], T is closed submodule. So that, by [Lemma (2.6)], T is St-closed submodule.

\section{Stc-M-INJECTIVE and Stc-self-INJECTVE MODULES}

Definition 3.1: Let $M$ and $A$ be $R$-modules. Then $A$ is called $S t c-M$-injective if every $R$ homomorphism $\varphi: T \rightarrow A$, where $T$ is St-closed submodule of $M$, can be extended to an $R$ homomorphism $\theta: M \rightarrow A$. An R-module A is called Stc-injective, if A is Stc-M-injective, for every $R$-module M. An R-module A is called Stc-quasi-injective or Stc-self-injective, if $A$ is Stc - A-injective.

\section{Remark and Examples 3.2:}

(1) It is easy to show that, if $A$ injective module then $A$ is also Stc-injective module.

(2) The converse of (1) is not true in general, as following example:

Let $A=Z_{2}$ as $Z$-module. Since $\mathrm{A}$ is the only St-closed submodule of $\mathrm{A}$, hence $\mathrm{A}$ is Stc-injective module. But, on other hand $\mathrm{A}=Z_{2}$ is not divisible $\mathrm{Z}$-module, $0=2 Z_{2} \neq Z_{2}$. Therefore, $Z_{2}$ is not injective.

3) Every semi-uniform (and hence every uniform) $R$-module is Stc-self-injective module.

Proof:- let A is semi-uniform R-module, then every submodule of $A$ is semi-essential submodule. So, that A is the only St-closed submodule in A. Therefore, A is Stc-self-injective.

(4) Every self-injective $R$-module is Stc-self-injective module.

(5) The converse of (4) is not true in general. Consider the module $Z$ as $Z$-module. It is well known that, $Z$ is not quasi-injective. But, since $Z$ is semi-uniform( in fact $Z$ is uniform), hence by (3), $\mathrm{Z}$ is Stc-self- injective. This show that, Stc-self-injective is proper generalization of quasiinjective.

(6) It is obvious that if $A_{2}$ is $A_{1}$-C-injective (and hence $A_{1}-C$-injective) then $A_{2}$ is also Stc- $A_{1}$ injective (this means that, Stc-injectivity is weak than c-injectivity). Thus we get the following implication:

Injective $\Rightarrow$ Quasi-injective $\Rightarrow R C$-quasi-injective $\Rightarrow C$-quasi-injective $\Rightarrow$ Stc-quasi-injective In the following proposition we will shows when the concepts Rc-injectivity, c-injectivity and Stc-injectivity are equivalents. 
Proposition 3.3: Let Mbe non-singular fully prime R-module and $A$ be any R-module. Then the following statements are equivalent:

(i) $A$ is rc-M-injective

(ii) $A$ is $c-M$-injective

(iii) $A$ is Stc $-M$-injective.

Proof: $(i) \Rightarrow$ (ii) $\Rightarrow$ (iii) It is clear.

(iii) $\Rightarrow$ (i) Suppose that, Ais Stc $-M$-injective. Let $\mathrm{K}$ be rationally closed submodule of $M$ with $\theta: K \rightarrow A$ be a homomorphism. Since $M$ be non-singular fully prime R-module, then by [proposition (2.8)], $\mathrm{K}$ is St-closed submoduleof $M$. So that, by $S t c-M$-injectivity of $A$, there is an R-homomorphism $f: M \rightarrow A$ such that extend $\theta$. This means, $A$ is $r c-M$-injective.

Corollary 3.4: Let Abe non-singular fully prime R-module. Then the following statements are equivalent:

(i) $A$ is rc-Self-injective

(ii) $A$ is $c$-Self-injective

(iii) $A$ is Stc-Self-injective.

In the following results we introduce some general properties of Stc $-M$-injectivity and Stc-self-injectivity.

Proposition 3.5:Let $K, T$ be two isomorphic $R$-modules and $M$ be any $R$-module. If $K$ is Stc $-M$-injective then $T$ is Stc $-M$-injective.

Proof: The proof is routine. $\square$

Proposition 3.6: Let Mand $A_{i}(i \in I)$ be R-modules. Then $A=\prod_{i \in I} A_{i}$ is Stc-M-injective if and only if $A_{i}$ is Stc $-M$-injective, for every $i \in I$.

Proof: First direction: Assume that, $\prod_{i \in I} A_{i}$ is $S t c-M$-injective. Let $\tau: T \rightarrow A_{i}$ be an $R$ homomorphism( where $\mathrm{T}$ is St-closed submodule of $M$ ). Now, consider the following mapping $\pi_{i}: \prod_{i \in I} A_{i} \rightarrow A_{i}$ and $\sigma_{i}: A_{i} \rightarrow \prod_{i \in I} A_{i}$ (where, $\pi_{i}$ is the natural projection of $\mathrm{A}$ into $A_{i}$ and $\sigma_{i}$ is the natural injection from $A_{i}$ into A, for every $i \in I$.). Since $\sigma_{i} \circ \tau$ is an R-homomorphism from $\mathrm{T}$ into $\mathrm{A}$ and we have $\mathrm{A}$ is $S t c-M$-injective then there exists an R-homomorphism $\theta: M \rightarrow A$ such that $\sigma_{i} \circ \tau=\theta \circ \mu($ where $\mu$ is inclusion map from $\mathrm{T}$ into $M$ ). Claim that, there exists an $\mathrm{R}$ homomorphism $f: M \rightarrow A_{i}$ such that $=f \circ \mu$, to show this, for every $t \in T, \tau(x)=I_{A_{i}} \circ f(x)=$ $\left(\left(\pi_{i} \circ \sigma_{i}\right) \circ \tau\right)(x)=\left(\pi_{i} \circ\left(\left(\sigma_{i} \circ \tau\right)(x)\right)=\left(\pi_{i} \circ((\theta \circ \mu)(x))=\left(\left(\pi_{i} \circ \sigma_{i}\right) \circ \mu\right)(x)=(f \circ \mu)(x)\right.\right.$ (where $I_{A_{i}}$ is identity mapping of $A_{i}$ for every $i \in I$ ). Therefore, this show that, $A_{i}$ is $S t c-M$ injective, for every $i \in I$.

Second direction: Assume that, $A_{i}$ is Stc $-M$-injective, for every $i \in I$. Let $g: T \rightarrow A=\prod_{i \in I} A_{i}$ be an $R$-homomorphism( where $\mathrm{T}$ is St-closed submodule of $\mathrm{M}$ ). Then, by assumption and for every homomorphism $\pi_{i} \circ g$ from $\mathrm{T}$ into $A_{i}$, there exists an R-homomorphism $\beta_{i}: M \rightarrow A_{i}$ such that $\pi_{i} \circ g=\beta_{i} \circ \mu$ thus by[ 9,Theorem (4.1.6)] there exists an R-homomorphism $\beta: M \rightarrow A$ with $\pi_{i} \circ \beta=\beta_{i}$. It is not hard to shoe that $g=\beta \circ \mu$. Therefore, $\prod_{i \in I} A_{i}$ is Stc $-M$-injective. As an immediate consequence of propositions (3.6) there is the following corollary.

Corollary3.7: Let $A=T \oplus T^{\prime}$. If $A$ is Stc $-M$-injective module, then $T$ is Stc $-M$-injective. 
Recall that an R-module $M$ is called chained, if for each sub modules A and B of $M$ either $A \leq B$ or $B \leq A[12]$.

Lemma3.8: [11]

(i) For a chained R-module M. If $A \leq$ Stc Band $B \leq$ Stc $M$, then $A \leq$ Stc $M$.

(ii)The intersection of two Stc-submodules is also Stc-submodule.

(iii)For a fully prime $R$-module $M$. If $(0) \neq A \leq$ Stc $B$ and $B \leq$ Stc $M$, then $A \leq$ Stc $M$.

Now, we are ready to consider the following results.

Proposition 3.9: Let $M$ be a chained $R$-module and $W$ be any $R$-module with $W$ is Stc-Minjective. Then the following statements hold.

(i) If $T \leq_{S t c} M$ then $W$ is Stc- $T$-injective.

(ii) If $T \leq_{S t c} M$ and $K \leq_{S t c} M$ then $W$ is Stc $-T \cap K$-injective.

Proof:((i) Let $T \leq_{S t c} M, L \leq_{S t c} T$ and $\varphi: L \rightarrow W$ be $R$-homomorphism. By [Lemma(3.8)(i)] we have $L \leq_{S t c} M$, hence by Stc $-M$ - injectivity of $W$, there exists a $R$-homomorphism $f: M \rightarrow W$ such that $f i_{T} i_{L}=\varphi$ where $i_{L}: L \rightarrow T$ and $i_{T}: T \rightarrow M_{1}$ are inclusion maps.Let $\beta=g \circ$ $i_{H}$. Clearly, $\beta$ is $R$-homomorphism, and $\beta=f i_{L}=f i_{T} i_{L}=\varphi$. Therefore, $W$ is Stc $-T$ injective.

(ii)It is clearly, follows from statement (i) and [Lemma(3.8)(ii)].

Corollary 3.10:Let $A_{1}$ and $A_{2}$ be any R-modules. If $A_{1} \oplus A_{2}$ is Stc-self - injective, then $A_{1}$ and $A_{2}$ are both Stc - self -injectives .

Corollary 3.11: An R-module Mis Stc-self-injective if $M$ is Stc - B-injective for every Stc-submoduleB of $M$.

Proof: It follows from proposition (3.9).

Proposition 3.12: A non-zero direct summands of Stc - self - injective fully prime R-module is Stc-self-injective.

Proof: Let $M$ be fully prime module with $\mathrm{M}$ is $S t c-$ self - injective and $(0) \neq T$ be any direst summand of $M$. Hence $M=T \oplus T^{\prime}$ for some submodule $T^{\prime}$ of $M$. Let $K$ be an Stc- submodule in $T$ and $f: K \rightarrow T$ be any $R$-homomorphism. Since, every direct summand is closed submodule then $\mathrm{T}$ is closed submodule in $\mathrm{M}$, hence by [Lemma (3.8)(iii)], $T$ is an Stc- submodule in $M$, implies $K$ is an Stc- submodule in $M$ then by Stc - self - infectivity of $M$, there exists $R$ homomorphism $\beta: M \rightarrow M$ such that $\beta i_{T} i_{k}=j_{T} f$, where $j_{T}$ is injection mapping from $T$ to $M$ and $i_{k}$ is inclusion from $K$ into $\mathrm{T}$. Let $\rho: M \rightarrow T$ be the projection map. Define $g: T \rightarrow T$ by $g(t)=\rho(\beta(t))$ for any $t \in T$. Follows that, for each $x \in K, g(x)=g i_{k}(x)=\rho \beta i_{K}(x)=$ $\rho j_{A} \circ f(x)=f(x)$. Hence $T$ isStc - self - injective. $\square$

Proposition3.13:Let $M$ be a chained $R$-module and $W$ be any $R$-module. WisStc-M-injective if and only if every direct summand $K$ of $W$ is Stc - T-injective for every Stc-submoduleT of $M$.

Proof: Suppose that, $W$ is $S t c-M$ - injective. Let $\mathrm{K}$ is a direct summand of $\mathrm{W}$ then by [Corollary (3.7)], $K$ is Stc $-M$-injective. Now, let $H$ is Stc-submodule of $\mathrm{T}$ then by [ Lemma 
(3.8)(i)], $H$ is Stc-submodule of $M$, hence it is clear that by [Proposition(2.9)], $K$ is Stc $-T$ injective. Conversely, it is directly from first direction, since we have $W$ is direct summand of $W$ and $M$ is Stc-submodule of $M$.

In general, not every Stc-submodule is direct summand[11]. For example, the submodule6 $Z$ is Stc-submodule of $Z_{12}$ as Z-module, but clear that, $6 Z$ is not direct summand of $Z_{12}$.

In the following results we show that when the Stc-submodule is direct summand

Proposition3.14:Let A be a Stc-self-injective R-module and let $T$ be an Stc-submodule of $A$ . If $T \cong A$ then $T$ is a direct summand of $A$.

Proof:In a similar way of [2], we can prove it.

In the following proposition, we give a characterization of $S t c-M$-injective modules.

Proposition3.15: Let $M=A_{1} \oplus A_{2}$ where $A_{1}$ and $A_{2}$ be two R-modules.Then the following statements are equivalent:

(i) $\quad A_{2}$ is Stc $-A_{1}$-injective

(ii) For every (St-closed) submoduleT of $M$ such that the intersection of $T$ with $A_{2}$ equal zero and $\pi_{1}(T)$ is St- closed submodule of $A_{1}\left(\right.$ where $\pi_{1}$ is the natural projection of $M$ into $A_{1}$ ), there exists a submodule $T^{\prime}$ of $M$ such that $T \leq T^{\prime}$ and $M=A^{\prime} \oplus A_{2}$.

Proof: $(i) \Rightarrow$ (ii) Assume that, $T$ be submodule of $M$ such that $T \cap A_{2}=0$ and let $A_{2}$ is Stc $-A_{1}$-injective. Let $\pi_{i}: M \rightarrow A_{i},(i=1,2)$ be the projective mapping and $\pi_{1}(T)$ is St-closed submodule of $A_{1}$. As $H \cap N_{2}=0$, the restriction of $\pi_{1}$ to $H$ is an $R$-isomorphism between $H$ and $\pi_{1}(T)$. Define $g: \pi_{1}(T) \rightarrow A_{2}$ such that $g(x)=\pi_{2}\left[\left(\pi_{1}\right)^{-1}(x)\right]$ (where, $\forall x \in \pi_{1}(T)$ ), $\exists t \in$ $T$ such that $\left.x=\pi_{1}(t)\right)$.It is easy to check that $\mathrm{g}$ is well-define and $\mathrm{R}$-homomorphism. Since $\pi_{1}(T) \leq_{S t c} A_{1}$ and $A_{2}$ is $S t c-A_{1}$-injective, then $g$ can be extended to an $R$-homomorphism $\theta: A_{1} \rightarrow A_{2}$. Define $T^{\prime}=\left\{t+\theta(t), t \in A_{1}\right\}$. Clearly, $T^{\prime}$ is a submodule of $M$ and $M=T^{\prime} \oplus A_{2}$. For every $t \in T$ we have $t=a_{1}+a_{2}$ where $a_{1} \in A_{1}$ and $a_{2} \in A_{2} \quad$.So, since $\pi_{1}(t)=a_{1}$ and $\pi_{2}(t)=a_{2}$ with $\theta\left(\pi_{1}(t)\right)=g\left(\pi_{1}(t)\right)=\pi_{2}(t)$ hence, we get $t=\pi_{1}(t)+\pi_{2}(t)=\pi_{1}(t)+$ $\theta\left(\pi_{1}(t)\right) \in T^{\prime}$. Therefore, $T$ is a submodule of $T^{\prime}$.

$(i) \Leftarrow(i i)$, Assume that, the statement (ii) holds. Let $W \leq_{S t c} N_{1}$ and let $\psi: W \rightarrow A_{2}$ be an $R$ homomorphism. Define $T=\{w-\psi(w), w \in W\}$. Clearly, $T$ is a sub module of $M$ such that $T \cap A_{2}=0$. It is easily to check that $\pi_{1}(T)=W$ and so that $\pi_{1}(T) \leq_{S t c} A_{1}$. Then by (ii), there exists a submodule $T^{\prime}$ of $M$ such that $T \leq T^{\prime}$ and $\quad M=T^{\prime} \oplus A_{2}$. Let $\pi_{2}: M \rightarrow A_{2}$ denote the projection with $\operatorname{ker} \pi_{2}=T^{\prime}$ and let $\phi: A_{1} \rightarrow A_{2}$ be the restriction of $\pi$ to $A_{1}$. For every $w \in$ $W, \phi(w)=\pi(w)=\pi[w-\psi(w)+\psi(w)]=\pi(w-\psi(w))+\pi(\psi(w))=\psi(w) \quad$ and, therefore, $\phi$ extends $\psi$.Thus $A_{2}$ is $S t c-A_{1}$-injective.

In the following, we characterize injective R-modules in terms of Stc-injectivity.

Proposition3.16:For an R-module A. Ais injective if and only if $A$ is $S t c-B$-injective for any $R$-module $B$.

Proof: (i) $\Longrightarrow$ (ii) It is clearly.

(ii) $\Rightarrow$ (i)As the same way of proof proposition (2.10). 
Recall an $R$-module $M$ be projective, if for each epimorphism $\theta: B \rightarrow A$ ( $\mathrm{A}$ and $B$ be any two $R$ module ) every $R$-homomorpism $\alpha: M \rightarrow A$ there is a homomorphism $\beta: M \rightarrow B$ with $\alpha=\theta \beta$ $[9, \mathrm{p} 117]$.

Theorem 3.17: Let $W$ be Stc $-B$-injective for any projective $R$-module .Then the following statements holed.

(i) $\frac{W}{T}$ is Stc $-B$-injective ( where $T$ is any submodule of $W$ ).

(ii) Any Stc-submodule of $B$ is projective

Proof: (i) $\Rightarrow$ (ii) Suppose that $T$ is any submodule of $W$ such that $\frac{W}{T}$ is $S t c-B$-injective. Now, Let $\mathrm{M}$ be injective $R$ - module and $\frac{M}{F}$ be any factor module of $\mathrm{M}$ with an $R$-epimorphism $: M \rightarrow \frac{M}{F}$ . Clearly that, $\frac{M}{F}$ is $S t c-B$ - injective. Suppose that $L$ is an Stc- submoduleof $B$, then by $B$-rcinjectivity of $\frac{M}{F}$, every $R$-homomorphism $\beta: L \rightarrow \frac{M}{F}$ can be extended to an $R$-homomorphism $\tau: B \rightarrow \frac{M}{F}$. Since $B$ is projective, then there exists an $R$-homomorphism $f: B \rightarrow M$ lifts $\tau$.Thus obviously, $\left.f\right|_{L}$ lifts $\beta$. This implies that $L$ is projective submodule of $\mathrm{B}$.

(ii) $\Rightarrow$ (i) Let $L$ is Stc- submodule of $B$, then $L$ is projective. Let $\frac{W}{T}$ and $W$ be two $R$-modules with an $R$-homomorphism $\tau: W \rightarrow \frac{W}{T}$. Consider $\rho: L \rightarrow \frac{W}{T}$ is an $R$-homomorphism and $W$ is Stc $-B$-injective. Then by projectivity of $L$, there exists an $R$-homomorpism $\theta: L \rightarrow W$ lifts $\rho$. Since $W$ is $S t c-B$-injective, then there exist an $R$-homomorphism $\beta: B \rightarrow W$ extends $\theta$. Thus, clearly $\theta \beta: B \rightarrow \frac{W}{T}$ extends $\rho$. $\square$

If every submodule of $M$ is a projective then the R-module A is called a hereditary module [7.]. Then the following result follows theorem (2.17).

Corollary 2.18:Let $A$ be a hereditary $R$-module. Then any factor $R$-module of an Stc $-A$ injective R-module is Stc - A-injective.

\section{REFERENCES}

[1] Abbas., M. S., and Ahmed, M.. A. (2011), "Rationally Extending and Strongly QuasiMonoform Modules", Al-Mustansiriya J. Sci. Vol.( 22), No( 3), pp.( 31-38).

[2] Abbas, M. S., and Nayef, M. S. (2015), "M-RC-injective and RC-quasi injective Modules", Int. J. of Math. Sci., Vol. (35) No. (2), PP. (1772-1779).

[3] Ali. S. M., and N. K. Abdullah,( 2009) " Semi-essential sub module and semi-uniform modules", J. of Kirkuk University-Scientific studies, Vol.(4) No. (1),.

[4] Behboodi M. Karamzadeh O. A. S, and Koohy H. (2004), "Modules whose certain sub module are prime", Vietnam J. of Mathematics, Vol. 32 No.(3): (303-317),.

[5] Clara, C. S., Some Generalizations of Injectivity, (1998), "Ph.D. dissertation", University of Glasgow.

[6] Dung, N.. V., Huynh, D. V.., Smith, P. F..\&Wisbauer., R.., (1994). "Extending Modules", Pitman Research Note Mathematics Series, Longman, Harlow.

[7] Dung N. V. and Smith, P. F.,(1992), "Hereditary CS-modules", Math. Scand. 71, (173180). 
[8] Goodearl, K.. R. (1976). "Ring Theory, Non-singular Rings and Modules", Marcel Dekker. Inc. New York.

[9] Kasch, F., (1982)," Modules and Rings", Academic Press Inc. London .

[10] Muna A. Ahmed, and Maysaa, R. Abbas, (2015). "On semi-essential sub modules", Ibn AlHaitham J. for Pure \& Applied Science, Vol. 28 (1), 179- 185.

[11] Muna A. A., and Maysaa, R. A., (2015)" St-closed sub modules ", Journal of Al-Nahrain University, Vol. 18 (3), (141- 149).

[12] Osofsky, B.L. (1991) "A construction of nonstandard uniserial modules over valuation domain". Bulletin Amer. Math. Soc25 : (89-97).

[13] Saymeh S. A.,( 1979) "on prime R-sub modules",Univ. Ndc. Tucuma'n Rev. Ser. A29, (129136).

[14] TiwariA.K.,et al.,(1979), "Generalization of Quasi-injectivity ", Progress of Math.,13:1.(3140). 